\title{
Moving Target Detection of Gaussian Mixture Model Based on Morphological Filter
}

\author{
Changhui Wang*a \\ School of Electronic Engineering \\ ${ }^{a}$ Tianjin Universitey of Technology and Education \\ Tianjin China
}

\author{
Jixiang Zhang ${ }^{\mathrm{b}}$ \\ School of Electronic Engineering \\ bTianjin University \\ Tianjin China
}

\begin{abstract}
The classical Gaussian background model is inadequate in the detection accuracy and speed. A novel algorithm is proposed for moving object detection based on mixture model which Gaussian mixture model and morphological filter are combined to find objects of interest that do not belong to the background. The algorithm performance is benchmarked using the video sequences created for the Background Models Challenge (BMC). For one thing, a mixture modeling algorithm is introduced to improve accuracy of detection. For another, morphological filter is used to suppress noise in the video frames, and the whole moving object is detected. What's more, experimental results show that the proposed algorithm has better performance compared with the classical Gaussian background modeling algorithms and can detect moving objects in various situations effectively.
\end{abstract}

Keywords-Gaussian Mixture Model; Object Detection; Morphological Filter

\section{INTRODUCTION}

In recent years, many scholars have proposed amounts of classic moving object detection algorithms [1]. Detecting object motion is a fundamental mission in video frame sequence analysis that is relevant to a widespread range of applications, for example, visual tracking tasks, video monitoring, surveillance of traffic and pedestrians and so on.

The common moving object detection includes the optical flow method, the frame difference method and the background subtraction method. Recently, the background modeling method based on statistical model has been developed for its fine adaptability to complex elements. To address the requirement for robustness and adaptation, many background modeling algorithms have been developed. Especially, Gaussian model algorithm has been adapted and improved by many researchers in all fields. GMMs were originally proposed by Friedman and Russell [2] to take up slow-moving objects. Many alternatives approach, including the following: saliency detection [3]; low-rank matrix factorization with iteratively reweighted least squares (IRLS) [4]; adaptive patch-based background modeling [5]; statistical modeling of the illumination effects [6]. In this paper, a new method is proposed by joint with Gaussian mixture model and morphological filter method. Our method can be better for moving object detection than conventional Gaussian model algorithm in the accuracy and speed of detection.

The rest of the paper is organized as follows: In Section 2, described Gaussian mixture model theory (Section 2.1) and morphological filter method can suppress the noise of the video frames (Section 2.2). Experimental results are shown in Section 3 , followed by conclusions in Section 4 .

\section{A. Gaussian Mixture Model}

\section{OBJECT DETECTION}

GMM algorithm claims the value of a particular pixel $\left(\mathrm{x}_{0}\right.$, $\left.\mathrm{y}_{0}\right)$ over time as a "random process", which is a time series of pixel value. It presents the recent history of each pixel $\left\{X_{1}\right.$, $\left.X_{2}, \cdots, X_{t}\right\}$ by mixture of $K$ Gaussian distributions, where $X_{t}$ is the pixel value of $\left(\mathrm{x}_{0}, \mathrm{y}_{0}\right)$ at time $\mathrm{t}$. The probability of observing the current pixel value is

$$
\mathrm{P}\left(\mathrm{X}_{\mathrm{t}}\right)=\sum_{\mathrm{i}=1}^{\mathrm{K}} \omega_{\mathrm{i}, \mathrm{t}} \cdot \eta\left(\mathrm{X}_{\mathrm{t},}, \mu_{\mathrm{i}, t}, \sigma_{\mathrm{i}, t}\right)
$$

Where ${ }^{H_{i}}$ is the mean value of the $i^{\text {th }}$ Gaussian in the mixture distribution at time t. ${ }^{\mathbb{W}_{\mathrm{it}}}$ is an estimate of the weight of the $i^{\text {th }}$ Gaussian in the mixture distribution at time $t,{ }^{M_{i t}}$ is the covariance matrix of the $i^{\text {th }}$ Gaussian in the mixture distribution at time $t$, and where $\eta$ is a Gaussian probability density function

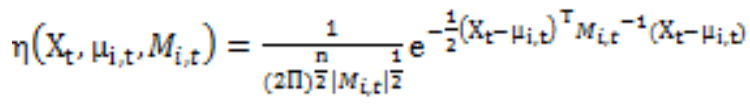

Where $\mathrm{n}$ is dimension of the $\mathrm{X}_{\mathrm{t}}$, the covariance matrix is assumed to be of the form $\left.M_{i t t}=\sigma_{i t}^{2}\right]$.This assumes is that the red, green, and blue pixel values are independent and have the same variances, where I is the unit matrix, ${ }^{2}{ }^{2}$ is the variance of the $\mathrm{i}^{\text {th }}$ Gaussian in the mixture distribution at time $\mathrm{t}$.

As time changes, the background in the scene also changes, resulting in increasing noise and systematic errors, therefore, the background needs to be updated in real time. For mixed Gaussian models, every Gaussian distribution model needs to be updated. For each pixel of the input current image, the pixel gray value of the current image is matched with the current background model according to the criterion (3) to determine whether the pixel obeys the existing Gaussian model

$$
R_{i, t}=\left\{\begin{array}{cc}
1 & \left|X_{t}-\mu_{i, t}\right| \leq D \sigma_{i t} \\
0 & \text { else }
\end{array}\right.
$$

Where D is confidence parameters. That is, the difference between the gray value of the current pixel and the average value ${ }^{\mu_{i}, t}$ of the $i^{\text {th }}$ Gaussian distribution is within d times of the variance $\sigma_{i, t}^{2}$ of the model. The value of $D$ mainly depends on the noise in the scene and usually takes between 2 and $3 . \mathbb{R}_{\mathrm{it}}$ is model matching operator, when it is 1 for the model which 
matched and 0 for the remaining models. The $\mu_{i, t}, \sigma_{i, t}^{2}$ for unmatched distributions remain the same.

Through the above judgment, if the current pixel value $X_{t}$ matches the $i^{\text {th }}$ Gaussian distribution, the weight of $i$ should be increased and the mean and variance of the model should be updated. In order to improved the accuracy and adaptability of the background model. Each Gaussian distribution model needs to be updated. First, update the model weights according to the following rules

$$
\omega_{i \mathrm{i}}=(1-\alpha) \omega_{\mathrm{it} t-1}+\alpha R_{\mathrm{ij} t}
$$

Where $\alpha$ is the learning rate, determines the speed of background updates. Parameters of the distribution which matches the new observation are updated as follows

$$
\left\{\begin{array}{l}
\mu_{\mathrm{i}, \mathrm{t}}=(1-\beta) \mu_{\mathrm{i}, \mathrm{t}-1}+\beta \mathrm{X}_{\mathrm{t}} \\
\sigma_{\mathrm{i} t \mathrm{t}}^{2}=(1-\beta) \sigma_{\mathrm{i}, \mathrm{t}-1}^{2}+\beta\left(\mathrm{x}_{\mathrm{t}}-\mu_{\mathrm{i}, \mathrm{t}}\right)^{\mathrm{T}}\left(\mathrm{x}_{\mathrm{t}}-\mu_{\mathrm{i}, \mathrm{t}}\right)
\end{array}\right.
$$

where $\beta$ is parameter learning rate, represented the Gaussian distribution of the parameters of the update speed, and $\beta=\alpha / \omega_{i j t}$. After the approximation, the weights are renormalized.

In order to measure the extent to which each Gaussian model represents a background model, sort K Gaussian models according to the size of the $\mathrm{P}={\sigma_{i}}_{\mathrm{i} t} / \sigma_{\mathrm{it}}$ value. The greater the correlation between the Gaussian model and the background, the smaller the variance ${ }^{\sigma_{i}^{2} t}$, and the greater the $p$ value, the more front sort. Therefore, the top row is the most likely to be a background model, otherwise small. Then the first B Gaussian models are chosen as the background model

$$
\mathrm{B}=\arg \min _{\mathrm{b}}\left(\sum_{\mathrm{i}=1}^{\mathrm{b}} \omega_{\mathrm{i} t \mathrm{t}} \geq T\right)
$$

where $\mathrm{T}$ is a threshold of the minimum number of models. The choice of $\mathrm{T}$ affects the detection accuracy and the amount of data to be computed. The current pixel matches with any of the former B Gaussian distributions, the point is the background point, otherwise, it is the sporting foreground point

\section{B. Morphological filter}

With the gradual deepening of theory and application of various branches of mathematics, nonlinear filtering represented by mathematical morphology has made remarkable progress in the protection of image edges and details. Morphological filter is an important non-linear filter which appears in recent years. It has been developed from the early binary filter to the later multi-value (grayscale) morphological filter. In shape recognition, edge detection and texture analysis, Image restoration and enhancement in areas such as a wide range of applications.
The basic idea of morphological operation is to use a predefined structural element to measure each pixel in a binary image. When the center point of a structural element coincides with a given pixel, a logical operation is performed on the intersection of the structural element and the image. , In order to judge the value of the pixel, the structure of the image is analyzed by the movement of the structural element, so as to achieve the purpose of recognizing and processing the image. Morphological filtering consists of four basic operations, which are expansion, erosion, closed, and open operations. Among them, the dilation and corrosion operations are the most basic operations of morphological filtering, while the open and closed operations are more advanced image processing based on the expansion and erosion.

Dilation operation: Dilation operation is the basic operation of morphological filtering. This method scans each pixel in the binary image, and takes the maximum of the sum of the image pixel and the structural element, that is, the structural element and the image coincidence area., use 0 or 1 to scan pixels. The dilation operation can be defined as follows:

$$
\mathrm{I} \oplus \mathrm{G}=\max _{(\mathrm{i}, \mathrm{j})}[\mathrm{I}(\mathrm{x}-\mathrm{i}, \mathrm{y}-\mathrm{j})+\mathrm{G}(\mathrm{i}, \mathrm{j})]
$$

Among them, I is a binary image to be processed, $\mathrm{G}$ is a structural element, usually represented by a matrix of 0 and 1 . Inflation is the process of expanding connected areas outwards. The degree of expansion is determined by the structural elements and is mostly used to fill voids inside the target object

Corrosion operation: Corrosion operation is a morphological filtering method with the opposite effect to the expansion operation. The basic idea is to scan each pixel in the binary image and take the minimum of the difference between the image pixel and the structural element, ie, the structural element. Corresponding to the image coverage area, use 0 or 1 to scan pixels. It is defined as:

$$
I \ominus G=\min _{(i, j)}[I(x+i, y+j)-G(i, j)]
$$

The corrosion is such that the boundary of the connected region can be contracted inward, and the manner and degree of contraction is controlled by the structural element $\mathrm{G}$. This operation can be used to eliminate isolated background noise and insignificant goals.

Morphological filtering takes full advantage of the geometric features and good algebraic properties of morphological operations, and mainly uses the open and close state of morphology to perform filtering operations. According to the basic principle of morphology, the morphological opening operation will remove the relatively bright distribution structure of the image which does not coincide with the shape of the structural elements, while keeping those coinciding parts; while the closing operation will fill those images with the structure Relatively dark distribution of elements that do not coincide, while retaining those that match. Since morphological operations perform image processing from the perspective of the geometry of the image, this excellent non-linear filter preserves the image structure from depassivation while filtering. 


\section{RESULTS AND DISCUSSION}

In this paper, our experiments are executed on windows $x p$ system with $3.00 \mathrm{GHz}$ Core 4 processor and $4.00 \mathrm{~GB}$ of memory. To analyze the robustness and effectiveness of the proposed algorithm, three experiments in different settings are demonstrated. The results are compared with adaptive GMM algorithm and the classical algorithm in subjective visual and objective parameters statistics.

Here is a comparison between the Gaussian mixture model method and our algorithm:

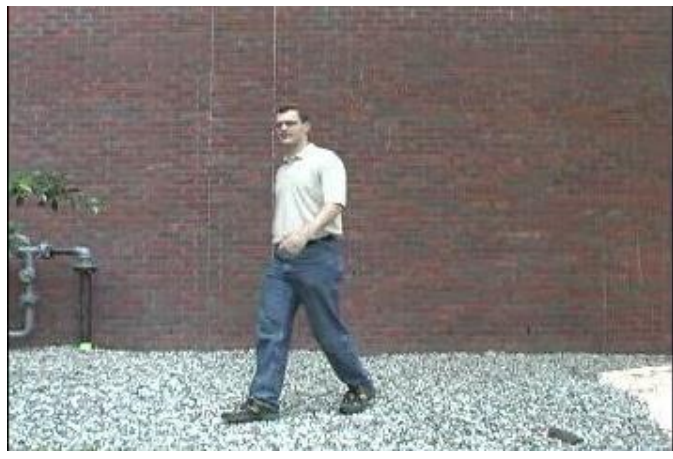

(a) Original frame of people

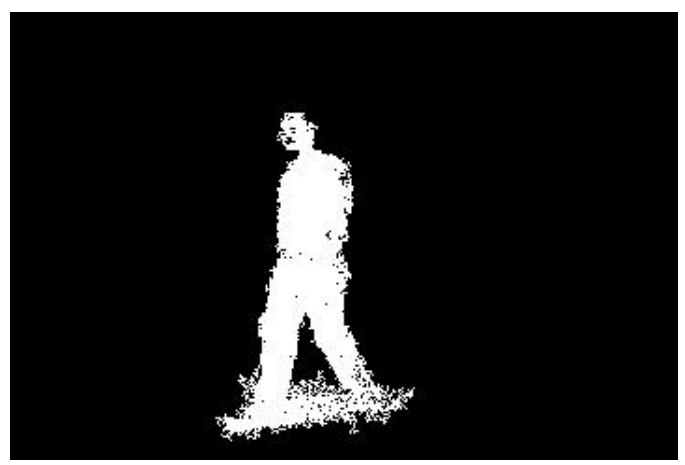

(b) our algorithm of people

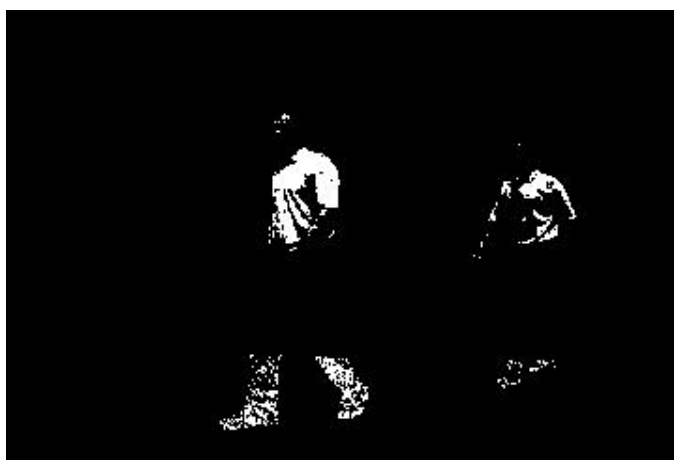

(c) GMM of people

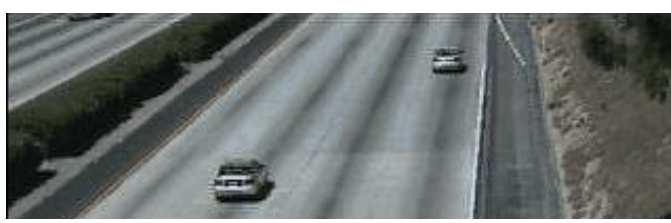

s

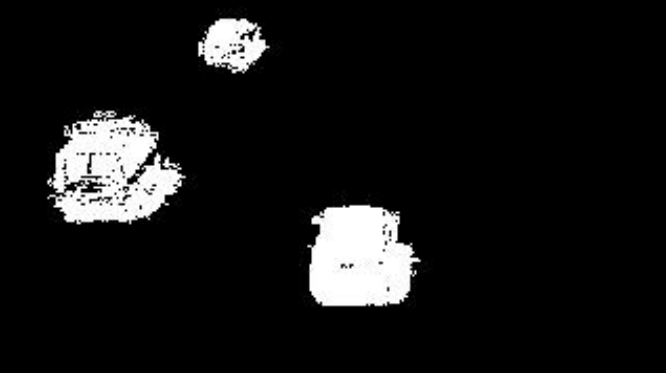

(e) our algorithm of cars

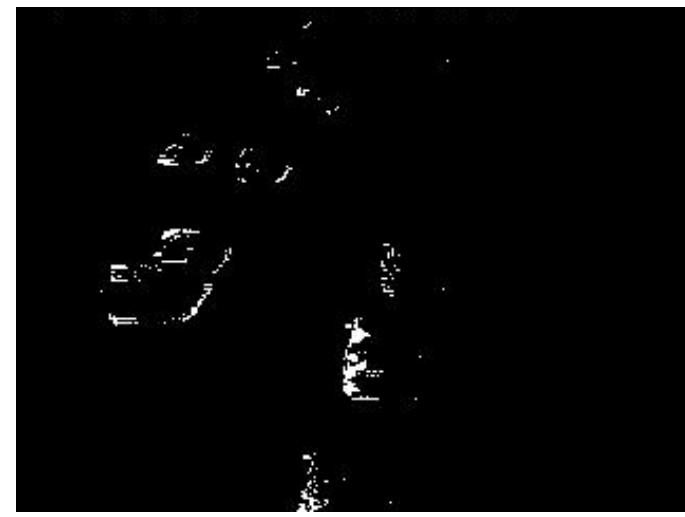

(f) GMM of cars

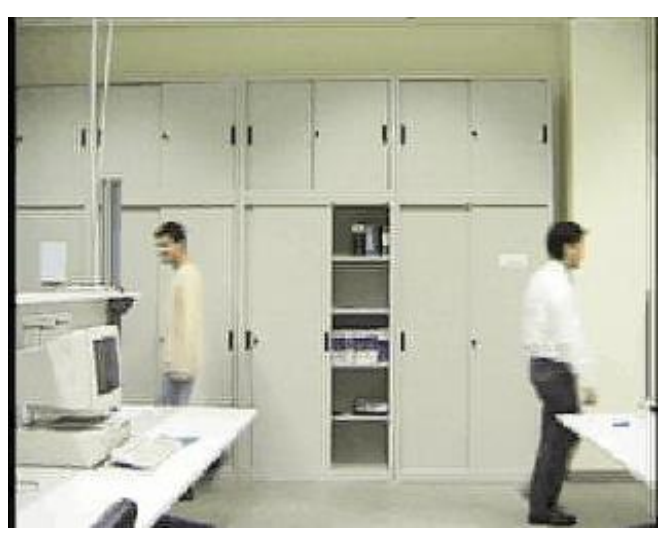

(g) Original frame of laboratory 


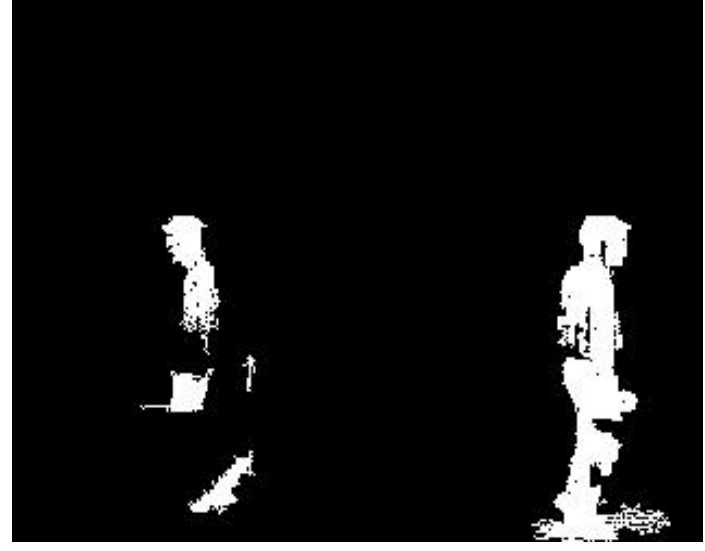

(h) our algorithm of laboratory

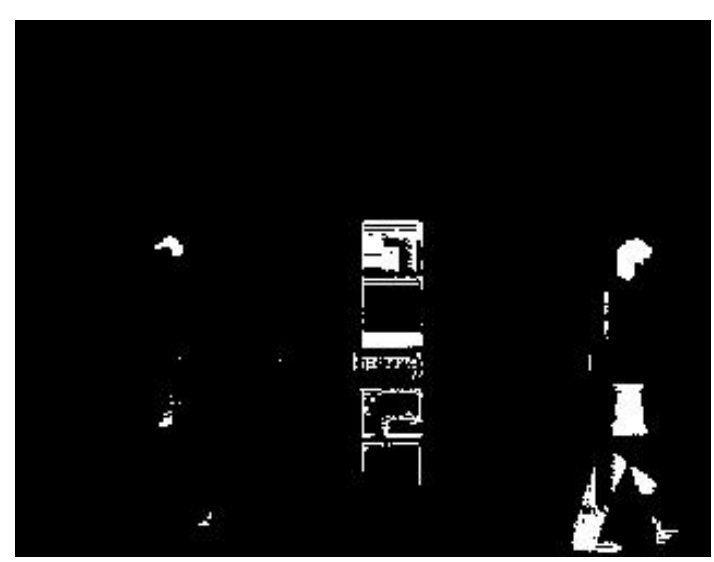

(i) GMM of laboratory

Fig. 1. The figure above shows the original image frame, the proposed algorithm and Gaussian mixture model test results, taken from the 57th frame, the 172 frame and the 578 frame.

The experimental results are shown in the images of Fig. 1, which are the results of the moving object detection. Figures (a), (d), and (g) are the original video frame images, and (b), (e), (h) are In this paper, the moving target detection results of the algorithm are shown in (c), (f), (i) are the results of the moving target detection based on the Gaussian mixture model. According to the indoor detection results of the 57th and 467th frame video images, the algorithm is not affected by the light conditions; the indoor and outdoor moving object detection results of the 172th and 578th frame video images can be obtained. The detection effect is better than outdoors, and it can be concluded that the detection result is not caused by the lighting factor.

The quantitative analysis of the detection rate of moving targets includes pixel level and object level. In this paper, object level is used to solve the moving target detection rate and false detection rate. The test results are shown in TABLE I. The following table shows the running frame rate of each video and TABLE II. Object level detection rate.
TABLE I. THE FOLLOWING TABLE SHOWS THE RUNNING FRAME RATE OF EACH VIDEO.

\begin{tabular}{|c|c|c|c|}
\hline algorithms & Video1(fps) & Video2(fps) & Video3(fps) \\
\hline GMM & 0.3003 & 0.2964 & 0.2972 \\
\hline $\begin{array}{c}\text { Our } \\
\text { algorithm }\end{array}$ & 0.88081 & 0.9066 & 1.0519 \\
\hline
\end{tabular}

TABLE II. OBJECT LEVEL DETECTION RATE.

\begin{tabular}{|c|c|c|c|c|c|}
\hline Video & $\begin{array}{c}\text { Target } \\
\text { numbers }\end{array}$ & $\begin{array}{c}\text { Number of } \\
\text { tests }\end{array}$ & $\begin{array}{c}\text { Number of } \\
\text { errors detected }\end{array}$ & $\begin{array}{c}\text { Detection } \\
\text { rate }\end{array}$ & $\begin{array}{c}\text { Error } \\
\text { rate }\end{array}$ \\
\hline $\begin{array}{c}\text { Video } \\
1\end{array}$ & 80 & 79 & 1 & $98.75 \%$ & $1.25 \%$ \\
\hline $\begin{array}{c}\text { Video } \\
2\end{array}$ & 500 & 491 & 9 & $98.20 \%$ & $1.80 \%$ \\
\hline $\begin{array}{c}\text { Video } \\
3\end{array}$ & 360 & 357 & 3 & $99.16 \%$ & $0.84 \%$ \\
\hline
\end{tabular}

Our experiments are based on indoor people walking and driving vehicles on the road as the goal, the detection of moving targets, the results show that our algorithm is significantly better than the traditional hybrid Gaussian model algorithm from the accuracy and rate of detection.

\section{CLUSION}

This paper proposes a novel moving object detection algorithm based on Gaussian mixture model and morphological filter method. The proposed algorithm could choose the number of components for each pixel. New algorithm dramatically enhances the convergence and the accuracy of background model, simultaneously maintaining the same temporal adaptability. In addition, we can effectively suppress the noise of the target image under the condition of preserving the details of the video image frame as much as possible remove the noise by using morphological filter. Comprehensive analysis of experimental results shows that our algorithm can detect moving objects in the different scenes effectively and has good robustness.

\section{REFERENCES}

[1] Bouwmans, T. (2014) Traditional and Recent Approaches in Background Modeling for Foreground Detection: An Overview. Computer Science Review, 11-12, 31-66.

[2] N. Friedman, S. Russell, Image segmentation in video sequences: a probabilistic approach, in: Proc. 13th Conf. on Uncertainty in, Artificial Intelligence, 1997.

[3] H.R. Tavakoli, E. Rahtu, J. Heikkilä, Temporal Saliency for Fast Motion Detection, Computer Vision - ACCV 2012 Workshops, vol. 7728, Lecture Notes in Computer Science, 2013, pp. 321-326.

[4] C. Guyon, T. Bouwmans, E. Zahzah, Foreground Detection Via Robust Low Rank Matrix Decomposition including Spatio-temporal Constraint, Computer Vision - ACCV 2012 Workshops, vol. 7728, Lecture Notes in Computer Science, 2013, pp. 315-320.

[5] V. Reddy, C. Sanderson, A. Sanin, B.C. Lovell, Adaptive patch-based background modeling for improved foreground object segmentation and tracking, in: Proc. of 7th Int. Conf. on Advanced Video and Signal Based Surveillance, 2010, pp. 172-179.

[6] J. Pilet, C. Strecha, P. Fua, Making background subtraction robust to sudden illumination changes, ECCV (2008) 567-580. 